FRI0629

APPLICATION OF AN ADVANCED NOISE REDUCTION ALGORITHM FOR IMAGING OF HANDS IN RHEUMATIC DISEASES - EVALUATION OF IMAGE QUALITY COMPARED TO STANDARD DOSE IMAGES

Katharina Ziegeler, Stefan Siepmann, Alexander Beck, Alexander Lembcke, Bernd Hamm, Kay Geert A. Hermann. Charité - Universitätsmedizin Berlin, Radiology, Berlin, Germany

Background: X-ray is the fundamental imaging technique in diagnosis and follow up of rheumatic diseases. As patients often require sequential $\mathrm{X}$-rays, dose reduction is of great importance. New advanced noise reduction algorithms allow for a dose reduction of up to $50 \%$.

Objectives: The aim of this study was to evaluate, whether the application of an advanced noise reduction algorithms is feasible in the context of imaging of rheumatic diseases.

Methods: A total of 298 patients were enrolled prospectively into three tiers: $80 \%, 64 \%$ and $50 \%$ dose reduction groups. All patients received imaging of one hand (laterality randomly assigned) with low-dose technique and of the contralateral side with standard protocol. All images were evaluated by two blinded independent readers who scored (on a scale of 1 to 5) the visualisation of bony cortex, trabeculae and joint spaces of fingers and wrist separately as well as soft tissue and overall contrast. Score values were analysed using T-tests for paired samples.

Results: Overall image quality (expressed by mean sum scores out of 40 ) of the $50 \%$ low-dose images was 31.52 (SD 1.94) vs. 31.66 (SD $1.82)$ for standard images $(p=0.217)$. Bony contours as well as trabeculae was equally well visualized in both image sets. An image example is given in Fig. 1 (Left hand: 50\%-dose image; right hand: standard-dose image). Soft tissue visualization was slightly lower for low-dose compared to standard images (mean score of 3.81 vs. $3.88 ; p=0.001$ ).

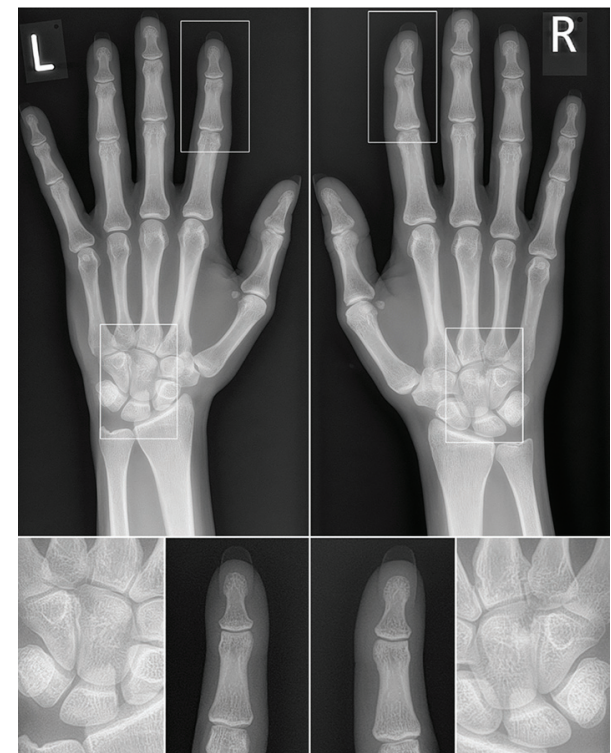

Conclusion: Overall image quality of low dose images was not inferior to standard dose images. Therefore, application of low-dose technology based on advanced noise estimation algorithms in the context of imaging of rheumatic diseases is feasible.

Disclosure of Interests: Katharina Ziegeler: None declared, Stefan Siepmann: None declared, Alexander Beck: None declared, Alexander Lembcke: None declared, Bernd Hamm Grant/research support from: Siemens, GE, Bayer, Samsung, Canon, Guerbet, Kay Geert A. Hermann Speakers bureau: AbbVie, MSD, Pfizer, UCB, Samsung DOI: 10.1136/annrheumdis-2019-eular.1922

\section{FRI0630 \\ DIAGNOSTIC UTILITY OF FLUORESCENCE OPTICAL IMAGING IN INDIVIDUALS WITH SUSPECTED ARTHRITIS - A PROBABILISTIC APPROACH}

Yogan Kisten ${ }^{1}$, Adrian Levitsky ${ }^{1}$, Hamed Rezaei ${ }^{1}$, Aase Hensvold ${ }^{1}$, Erik Af Klint ${ }^{1}$ Ronald van Vollenhoven ${ }^{1,2}$, Anca Catrina ${ }^{1} .{ }^{1}$ Karolinska Institute, Department of Medicine, Division of Rheumatology and Early Arthritis Clinic of the Karolinska University Hospital, Stockholm, Sweden; ${ }^{2}$ Amsterdam Rheumatology and immunology Center, Amsterdam, Netherlands

Background: In the arsenal of available techniques used for arthritis pre diction and diagnosis ${ }^{1}$, fluorescence optical imaging (FOI) has been proven useful in detecting clinically manifest and silent synovitis of the hands and wrists of patients with various rheumatic diseases, particularly rheumatoid arthritis (RA) ${ }^{2}$

Objectives: To assess the diagnostic utility of $\mathrm{FOI}$ in individuals with joint symptoms referred for further rheumatologic investigation, using a probabilistic approach.

Methods: Those with increased suspicion of inflammatory arthritis were referred to the rheumatology unit and clinic of Karolinska University Hos pital. On acquiring informed consent and medical history, the standard clinical examination coupled with ultrasound findings of fingers, wrists and feet were assessed. Laboratory results included ESR, CRP, RF, and ACPA tests. Using the above information, a diagnostic probability assessment was completed by the responsible rheumatologist, where the probability of a) any inflammatory joint disease and b) rheumatoid/RA was given on a 5-point scale - ranging from unlikely $(0-20 \%)$ to very likely $(80-100 \%)$ probability. Subsequently, an FOI examination was performed After reviewing the image reports in consensus, post-FOI diagnostic probabilities were again scored, using the same scale. If no score change in probability resulted, the rheumatologist was asked to mark whether FOI was still helpful in the diagnostic decision-making. Proportions of individuals with maximal and minimal diagnostic certainty pre- and post-test were compared using Fisher exact tests, and one-sample binomial tests for assessing the helpfulness of $\mathrm{FOI}$ in the absence of pre- and post-test probability score changes.

Results: Of 24 individuals screened, 21 without prior rheumatic diagnosis were included $(66.7 \%$ female, $11 \mathrm{RF}(+), 10$ ACPA (+), with age average and symptom duration (SD) of $55.6( \pm 18.1)$ years and $13.9( \pm 15.3)$ months respectively). The final diagnoses were: early $R A(n=17)$, other inflammatory joint disease $(n=3)$, and non-inflammatory joint disease $(n=1)$. Regarding diagnosis of any inflammatory arthritis, where the proportion of patients for whom diagnostic certainty was maximal - namely, combining $<20 \%$ (lowest probability) or $>80 \%$ (highest probability) of diagnostic likelihood - there was an increase from 52.4\% ( $n=11 / 21)$ maximal certainty before $\mathrm{FOI}$ to $80.1 \%$ (17/21) maximal certainty after FOI ( $\mathrm{p}=0.035$ ) Regarding early RA, the maximal diagnostic certainty increased from $57.1 \%(12 / 21)$ to $71.4 \%(15 / 21) \quad(p=0.002)$, respectively. In the event that diagnostic certainty scores didn't change pre- vs. post-test (15/21 cases, any inflammatory joint disease; $13 / 21$ cases, early RA), the diagnosing rheumatologist indicated that FOI was still helpful in setting a final diagnosis for most cases $(86.7 \% \quad(13 / 15) \quad p=0.007 ; 84.6 \% \quad(11 / 13), p=0.022$ respectively).

Conclusion: FOI significantly increased the diagnostic certainty and confidence of rheumatologists in establishing the presence and absence of inflammation in individuals suspected of inflammatory arthritis. The changes from pre- to post-test quantify the diagnostic utility of FOI in probabilistic terms.

\section{REFERENCES:}

[1] Rezaei H, Torp-Pedersen S, Af Klint E, et al. 2014 Diagnostic utility of musculoskeletal ultrasound in patients with suspected arthritis - A probabilistic approach. Arthritis Res Ther 2014;16:448. doi.101186/s13075-0140448-6

[2] Kisten Y, Györi N, af Klint E, et al. 2015 Detection of clinically manifest and silent synovitis in the hands and wrists by fluorescence optical imaging. RMD Open 2015;1: e000106. Doi: :10.1136/rmdopen-2015-000106 https://www.ncbi.nlm.nih.gov/pubmed/26535142

Disclosure of Interests: Yogan Kisten: None declared, Adrian Levitsky None declared, Hamed Rezaei: None declared, Aase Hensvold: None declared, Erik Af Klint: None declared, Ronald van Vollenhoven Grant research support from: AbbVie, BMS, GSK, Pfizer, UCB, Consultant for: AbbVie, AstraZeneca, Biogen, Biotest, BMS, Celgene, Gilead, GSK, Jans sen, Lilly, Novartis, Pfizer, UCB, Speakers bureau: AbbVie, Lilly, Pfizer, UCB, Anca Catrina Grant/research support from: Yes, but not for the presented study.

DOI: 10.1136/annrheumdis-2019-eular.2810 